J. Dairy Sci. 99:8891-8900

http://dx.doi.org/10.3168/jds.2016-11210

(C) 2016, THE AUTHORS. Published by FASS and Elsevier Inc. on behalf

of the American Dairy Science Association ${ }^{\circledR}$. This is an open access article under

the CC BY-NC-ND license (http://creativecommons.org/licenses/by-nc-nd/3.0/).

\title{
Heritability and genome-wide association mapping for supernumerary teats in French Alpine and Saanen dairy goats
}

\author{
Pauline Martin, ${ }^{1}$ Isabelle Palhière, Gwenola Tosser-Klopp, and Rachel Rupp \\ Génétique, Physiologie et Systèmes d'Elevage (GenPhySE), Université de Toulouse, INRA, INstitut Polytechnique de Toulouse (INPT), \\ Ecole Nationale Vétérnaire de Toulouse (INP-ENVT), Castanet Tolosan, France
}

\begin{abstract}
This paper reports a quantitative genetics and genomic analysis of undesired presence of supernumerary teats (SNT) in goats. Supernumerary teats are a problem in goat breeding as they can considerably impede machine milking efficiency, leading to increased milking time and injury. This phenotype has routinely been recorded for the past $15 \mathrm{yr}$ in French Alpine and Saanen goats. Around $4 \%$ of the females had been assigned the SNT phenotype and consequently could not be included in the breeding program as elite animals. The heritability of this binary trait, estimated by applying linear logistic polygenic models to 32,908 Alpine and 23,217 Saanen females, was 0.40 and 0.44, respectively. A genome-wide association study was implemented using a daughter design composed of 810 Saanen goats sired by 9 artificial insemination bucks and 1,185 Alpine goats sired by 11 bucks, genotyped with the goatSNP50 chip (Illumina Inc., San Diego, CA). This association study was based on logistic polygenic models, one with separately taken single nucleotide polymorphisms and the other with haplotypes as fixed effects. The 2 breeds were analyzed together and separately. No region was found to be significant at the genome level, but 17 regions on 10 chromosomes were significant at the chromosome level. These signals were always only slightly above the chromosome significance threshold and only a few of them overlapped across analyses. No evidence of segregation of a major gene in our Saanen and Alpine populations was observed, suggesting that SNT presence is inherited in a polygenic fashion. This conclusion regarding SNT determinism agrees with recent association analyses in cattle, and one locus was even found in an orthologous region. The possibility of applying markers-based selection on the SNT trait is therefore unlikely, but, as this trait is heritable and
\end{abstract}

Received March 23, 2016.

Accepted July 6, 2016.

${ }^{1}$ Corresponding author: pauline.martin@toulouse.inra.fr routinely recorded, it could be managed by attributing a dedicated estimated breeding value.

Key words: supernumerary teat, goat, genome-wide association study

\section{INTRODUCTION}

Supernumerary teats (SNT or polythelia) are common in many mammalian species. The frequency of SNT varies considerably between species and even between breeds. In cattle, SNT frequencies of 15 to $69 \%$, depending on the breed and geographical location, are reported in the literature (Brka et al., 2002). In a study of 398 Turkish Saanen goats, all from the same experimental herd (Brka et al., 2007), the frequency of affected animals was $17 \%$.

In pigs, a high number of functional teats is beneficial to respond to the increase in litter size (Chalkias et al., 2013). In other species, especially dairy ruminants, SNT are more commonly considered as defects. They are usually located on the embryonic mammary line (Urbani and Betti, 1998) and, in cattle, most often in a caudal position to the normal teats (Gifford, 1934; Rowson et al., 2012). They may connect to the sinus of another teat, have a separate supernumerary gland, or have no gland at all or an abortive one (Gifford, 1934; Blowey and Weaver, 2011). Therefore, SNT can be functional (i.e., with emission of milk) or not; SNT are not restricted to females and can also be observed in males.

Environmental factors predisposing for SNT are still not well known but a role of intrauterine hormones has been suggested (Brka et al., 2002). As SNT are formed during embryogenesis, the environmental effects result from the intrauterine environment and, more broadly, from the dam's environment. In female pigs, the teat number is related to the proportion of males in the litter (Drickamer et al., 1999), and this is probably due to prenatal hormones and the intrauterine position of the fetus. In cattle, a higher incidence of SNT has been reported in cows born from multiparous animals than in cows born in first parity, when the dam is simply 
pregnant and not lactating at the same time (Brka et al., 2002). This difference could be due to differential expression of the genes in the Wnt signaling pathway, which may be involved in abnormal teat development (Pausch et al., 2012).

A genetic basis for the presence of SNT has been hypothesized. In cattle, the estimated heritability ranged from 9 to $63 \%$, with most estimates being around $40 \%$ (Brka et al., 2002). In goats, the single estimate of heritability, based on a small sample of animals, was 0.34 (Brka et al., 2007).

The pattern of inheritance has been studied in different species. A simple dominant or recessive inheritance has been hypothesized in humans (Urbani and Betti, 1996), sheep (Vainikainen, 1945), and guinea pigs (Goertzen and Ibsen, 1951). In cattle, the inheritance pattern seems to be more complex, with some evidence of incomplete penetrance or an oligo- or polygenic determinism (Brka et al., 2000, 2002). Some QTL mapping has been carried out for teat number in cows (Pausch et al., 2012; Joerg et al., 2014) and pigs (Hirooka et al., 2001), but, to our knowledge, no study of the genetic architecture of SNT in goats has been published so far.

Udder morphology is very important in dairy ruminants, as it affects how the milking machine is plugged on the individuals (Labussière, 1988; Peris et al., 1996). The existence of SNT can considerably impede machine milking efficiency, leading to increased milking time and injury. Moreover, SNT and glands may act as reservoirs for bacteria and be responsible for increased susceptibility to mastitis (Brka et al., 2000).

The aim of the current study was to estimate the genetic parameters governing SNT presence and then detect the genome regions governing the trait by performing a genome-wide association study (GWAS) based on goatSNP50 chip (Illumina Inc., San Diego, CA) data. Those analyses could determine the feasibility of genetic or genomic selection against SNT in the French Alpine and Saanen dairy goat breeds.

\section{MATERIALS AND METHODS}

\section{Ethics Statement}

The DNA samples for this study came from France and were stored at the Laboratoire d'Analyses Génétiques pour les Espèces Animales (Jouy en Josas, France; www.labogena.fr). No sperm collection or blood sampling was performed specifically for this study. Sperm was collected by Capgenes (Mignaloux Beauvoir, France; http://www.capgenes.com/) from bucks at the AU stations, with authorization (FR CC 860) from the Direction Générale de l'Alimentation (Paris, France), and we used extra doses from this collection.
Blood samples were taken at commercial farms. The animals were not part of any experimental design but were sampled by veterinarians or under veterinarian supervision for routine veterinary care; extra samples were requested when blood sampling took place.

\section{Population Resources and Phenotypes}

The pedigrees and phenotypes in our study were taken from the French national genetic database used for official genetic evaluations. The animals were Saanen and Alpine goats involved in the national breeding program.

The phenotype frequency and heritability estimates were determined from all the females evaluated by the goat breeding organization, Capgenes, with their 2 parents known and born between 2005 and 2007. Flocks with fewer than 10 animals were excluded. As some breeders absolutely want to avoid animals with SNT in their lactating herds, and consequently eliminate animals born with SNT at a young age before any recorded phenotyping, flocks with no animal carrying SNT were removed to avoid bias. Finally, 23,217 Saanen and 32,908 Alpine goats from 532 different flocks were included in the data set.

The GWAS data set came from a subset of Saanen and Alpine goats involved in a daughter design implemented in 2009 and 2010 as part of the national Genomcap (France) and the European Union 3SR (www.3srbreeding.eu) projects. The design consisted of a total of 2,254 goats, from 250 commercial farms, that were sired by 20 different AI bucks. The 20 bucks were selected, among the AI sires available in 2009 and 2010, to be both representative of the genetic diversity of the total population and to maximize the genetic diversity between families. There were 9 Saanen and 11 Alpine families.

Animals from these 2 data sets were phenotyped during the first or second lactation by official classifiers from the breeders' association (Capgenes). The information about SNT was qualitative (i.e., presence or absence); none of the 20 sire bucks possessed SNT. Functional and nonfunctional SNT were not distinguished in our study.

\section{Genetic Parameter Estimation}

Phenotype frequencies were calculated separately for Alpine and Saanen breeds, using SAS ver. 9.2 software (SAS Institute Inc., Cary, NC). The phenotype frequencies in the data set used for the GWAS were also calculated to compare this sample with the total population. The different frequencies were compared 
by chi-squared tests, and significance differences were declared at the $5 \%$ threshold.

\section{Estimation of Heritability}

Variance components for SNT presence were estimated separately in the 2 breeds with an animal binary logistic mixed model using ASReml 3.0 Software (Gilmour et al., 2009). The general logistic model form was:

$$
\operatorname{Logit}(p)=\mathbf{W b}+\mathbf{Z} a+e,
$$

where $\operatorname{Logit}(p)=\ln \frac{P(Y=1)}{1-P(Y=1)}$ and $P(Y=1)$ denotes the probability of a goat to have a SNT as performance $Y$, with the vector of fixed effects $\mathbf{b}$, including flock of birth and year of birth; $a$ is the random genetic animal effect, where $a$ is assumed to follow $a \sim N\left(0, \mathbf{A} \sigma_{a}^{2}\right)$ and $\mathbf{A}$ is the relationship matrix based on pedigree information; $\mathbf{W}$ and $\mathbf{Z}$ are the incidence matrices; and $e$ is the residual effect.

The pedigree files consisted of 4 generations including 62,470 and 87,989 animals in the Saanen and Alpine breeds, respectively. Trait heritability was estimated from the ratio of the animal variance component to the sum of the animal variance component and the residual variance.

\section{GWAS}

Quality Control of SNP. All 2,254 goats and 20 bucks were genotyped using the Illumina goatSNP50 chip (Tosser-Klopp et al., 2014). The DNA extraction from blood samples and genotyping were performed at Laboratoire d'Analyses Génétiques pour les Espèces Animales. Quality control was performed across breed and included the SNP call rate $(>99 \%)$, animal call rate $(>98 \%)$, minimum allele frequency $(>2 \%)$, Hardy Weinberg equilibrium ( $P$-value above $10^{-6}$ ), and pedigree inconsistency removal. After these controls, 810 Saanen goats (including 55 animals with SNT) and 1,175 Alpine goats (including 51 animals with SNT) and their 20 sires remained. After editing, a total of 49,647 out of 53,347 synthesized SNP were validated for further analyses. The marker order and positions were based on the caprine Assembly CHIR_1.0 (http:// www.ncbi.nlm.nih.gov/assembly/GCF_000317765.1) and can be downloaded (http://www.goatgenome.org/ data/capri4dbsnp-base-CHI-OAR-UMD.csv).

Population Stratification Assessment. Population stratification was assessed by calculating the genetic relationship matrix from all SNP, and the 2 principal components, using the GCTA software (Yang et al., 2011). The first principal component values were plotted against the second principal component values to identify potential clustering among animals with or without SNT.

$\boldsymbol{G} \boldsymbol{W A} \boldsymbol{S}$. Genome-wide association study was carried out for each breed separately and together. Two models were used in each case. The first model was an SNP GWAS: the effect of each SNP was tested individually from the following logistic model with mixed effects:

$$
\operatorname{Logit}(p)=\mathbf{W} \mathbf{b}+\mathbf{Z} a+e,
$$

where $\operatorname{Logit}(p)=\ln \frac{P(Y=1)}{1-P(Y=1)}$ and $P(Y=1)$ denotes the probability of a goat to have a SNT as performance $Y ; \mathbf{b}$ is the vector of fixed SNP effect [genotype expressed with 0 (homozygous), 1 (heterozygous), or 2 (the other homozygous)]; $\mathbf{W}$ is the incidence matrix of b corresponding SNP effect to individuals; $a$ is the random genetic animal effect (polygenic effect) where $a$ is assumed to follow $a \sim N\left(0, \mathbf{A} \sigma_{a}^{2}\right)$ and $\mathbf{A}$ is the relationship matrix based on pedigree information; $\mathbf{Z}$ is the incidence matrix accounting for pedigree relationship structure among individuals; and $e$ is the random residual effect following $e \sim N\left(0, \mathbf{I} \sigma_{e}^{2}\right)$, where $\mathbf{I}$ is the identity matrix and residual variance was set to 1 . Only the 4 most recent generations of the pedigree were used in this analysis.

These calculations were done with restricted maximum likelihood using ASReml Software (Gilmour et al., 2009). Each calculated SNP effect was subjected to a $t$-test to calculate the $-\log _{10}(P$-value $)$. Because of the test multiplicity, a Bonferroni correction of $\alpha=5 \%$ was applied for both genome-wide and chromosomewide thresholds (Significance threshold $=\alpha$ /number of SNP). Any SNP with $P<1.04 \times 10^{-6}$ were considered to be significantly associated at the genome-wide level. The chromosome-wide significance thresholds ranged from $1.62 \times 10^{-5}$ to $6.34 \times 10^{-5}$ depending on the chromosome size.

The second model used haplotypes; haplotypes were constructed using Phasebook (Druet and Georges, 2010). The method implemented in the Phasebook software package was designed to obtain phased haplotypes in a population with high relationships, by the successive action of different software: haplotypes were first reconstructed based on familial information (Mendelian segregation rules and linkage information) using LinkPhase; and then the gaps between the reconstructed elements were filled in by applying a hidden Markov model from the combined action of Beagle (Browning 
and Browning, 2007) and DAGPHASE (Druet and Georges, 2010). DAGPHASE was then run to attribute randomly missing alleles before the use of Beagle, which, used iteratively with DAGPHASE, constructed optimal directed acyclic graphs and improved those graphs at each iteration. The final haplotype information output from the process were haplotype hidden states defined for each SNP position.

The following parameters were used: the threshold probability value to attribute parental origin was set at 1.0 for LinkPHASE as recommended by Druet and Georges (2010). The parameters of iteration and shift from Beagle (version 3.3.2) were set to 10 and 0.0 respectively, according to the instruction from Browning and Browning (2007). Different scale parameters were tested and scale $=6$ was chosen for its fit on data according to the quantile-quantile (QQ) plots. The maximum number of haplotype hidden states at one position were 10 in Saanen 12 in Alpine, and 15 when both breeds were analyzed together.

The model used for this haplotype-based association was identical to the first model except that the haplotype effect (i.e., the hidden cluster effect), was fitted instead of an SNP in the model:

$$
\operatorname{Logit}(p)=\mathbf{W} \boldsymbol{\beta}+\mathbf{Z} a+e,
$$

where $\operatorname{Logit}(p)=\ln \frac{P(Y=1)}{1-P(Y=1)}$ and $P(Y=1)$ denotes the probability of a goat to have SNT as performance $Y ; \boldsymbol{\beta}$ is the vector of haplotype effect (hidden cluster effect); $\mathbf{W}$ is the incidence matrix of $\boldsymbol{\beta}$ corresponding haplotype effect to individuals (the 2 haplotypes per animal and position were fitted as 2 related fixed effects so that the same haplotypes provided the same effects); $a$ is the random genetic animal effect (polygenic effect); and $\mathbf{Z}$ and $e$ are the same as in model [2]. The same pedigree was used throughout.

Restricted maximum likelihood was applied to solve the model using ASReml (Gilmour et al., 2009). The haplotype effects of all haplotypes were calculated, an F-test was performed to determine significance and a Bonferroni correction was applied to $\alpha=5 \%$.

Validation of Test Statistics with $Q Q$ Plots. The QQ plots were constructed for each analysis (GWAS model by population combination) to check the general distribution of the test-statistics and to assess the degree of fit of the model for this analysis. Under the hypothesis that most SNP are not associated with the trait, the corresponding QQ plots should follow the $45^{\circ}$ line $\mathrm{y}=\mathrm{x}$ to confirm the good fit of the observed-toexpected (theoretical) distributions. This was verified for the QQ plots obtained here (Figure 1).

\section{RESULTS}

\section{Frequency of the SNT Phenotype in French Saanen and Alpine Goats}

The distribution and frequencies of the SNT phenotype in the different data sets are presented in Table 1. Frequencies were close to $4 \%$ and similar between years and breeds, except for the GWAS data set for the Saanen breed, which exhibited a slightly and significantly higher frequency $(6.8 \%, P=0.0008)$. In addition, the SNT frequency varied considerably between families in the GWAS data set, ranging from 0.8 to $11.9 \%$, as shown in Figure 2.

\section{Heritability}

The estimated heritability of SNT presence in the 2 breeds was similar; that is, $0.44(\mathrm{SE}=0.04)$ and 0.40 $(\mathrm{SE}=0.04)$ in Saanen and Alpine, respectively, with genetic variances of 0.77 and 0.66 , respectively.

\section{Population Stratification Assessment}

Results from a principal component analysis based on SNP information were then used to construct and plot the population structure and investigate the possibility of improving subsequent analyses. As illustrated in Figure 3, the population structure reflects the presence of 2 breeds (Alpine on left and Saanen on right) and the 20 families used in our daughter design. Figure 3 shows the broad distribution of the studied phenotype on the graph. As the GWAS used a relationship matrix to take family structure into account, no additional correction was made for population stratification.

\section{GWAS Analyses}

The GWAS were conducted within and across breeds and using SNP or haplotypes. The corresponding inflation factors range from 1.02 to 1.05. Manhattan plots are shown in Figure 4. These 6 analyses did not indicate any significant region at the genome threshold, but 17 regions were significant at the chromosome level. All those latter significant positions are detailed in Table 2. One to 4 chromosome-wide significant SNP were found in each analysis. Only the locus found on Capra hircus autosome (CHI) 12 around $25 \mathrm{Mb}$ overlapped (within a 5 -Mb interval) across 2 different analyses (Saanen haplotype analysis at $28.7 \mathrm{Mb}$ and 2 breeds haplotype analysis at $24.2 \mathrm{Mb}$ ). Thus, different chromosome-wide significant regions were found in each breed and mostly new hits were found when the data sets from the 2 breeds were combined. 
Table 1. Proportion (and number) of females phenotyped as supernumerary teats (SNT) according to year of birth in the Saanen and the Alpine breeds recorded in the national database and born in 2005, 2006, or 2007

\begin{tabular}{|c|c|c|c|c|c|}
\hline \multirow[b]{2}{*}{ Item $^{1}$} & \multicolumn{4}{|c|}{ National population (phenotyped) } & \multirow{2}{*}{$\begin{array}{c}\text { GWAS } \\
\text { population }\end{array}$} \\
\hline & Birth year 2005 & Birth year 2006 & Birth year 2007 & Total & \\
\hline Saanen & $\begin{array}{r}3.9 \\
(286)\end{array}$ & $\begin{array}{c}4.3 \\
(335)\end{array}$ & 4.0 & 4.1 & $\begin{array}{r}6.8 \\
(55)\end{array}$ \\
\hline Alpine & $\begin{array}{c}4.3 \\
(427)\end{array}$ & $\begin{array}{c}4.3 \\
(461)\end{array}$ & $\begin{array}{c}4.1 \\
(499)\end{array}$ & $\begin{array}{c}4.2 \\
(1387)\end{array}$ & $\begin{array}{c}4.3 \\
(51)\end{array}$ \\
\hline
\end{tabular}

${ }^{1}$ The frequency and number obtained for each breed in the genome-wide association study (GWAS) population is shown for comparison.

\section{DISCUSSION}

The frequency of SNT in the French dairy goat population, as recorded in the national database, is very low (average of $4 \%$ ). The true frequency is probably higher as the trait is not easy to phenotype. Indeed, some technical recommendations advise cutting any visible SNT just after birth and as some breeders may do this, subsequent phenotyping can be erroneous. Breeders are also likely to avoid SNT carriers when they choose young females for replacement. A proportion of affected animals will therefore no longer be present in the flocks when the females are phenotyped by classifiers during their first lactation. The frequencies observed here are indeed lower than those reported in the literature [i.e., $17 \%$ for goats (Brka et al., 2007) or between 15 and $69 \%$ for cattle (Brka et al., 2002)]. The goat data in the literature were obtained from a single experimental herd and may not be representative of the Turkish Saanen goat population.

The heritability estimates in this study $\left(\mathrm{h}^{2}=0.40\right.$ to 0.44 ) are within the range found for the same trait in the literature; that is, similar to the most frequent results in cows, with $\mathrm{h}^{2}=45 \%$ for German Simmental and $\mathrm{h}^{2}=43 \%$ for German Brown Swiss (Brka et al., 2002), and slightly but not significantly different to the single published goat study, where $\mathrm{h}^{2}$ was $34 \%$ (Brka et al., 2007), given confidence intervals of estimates. We
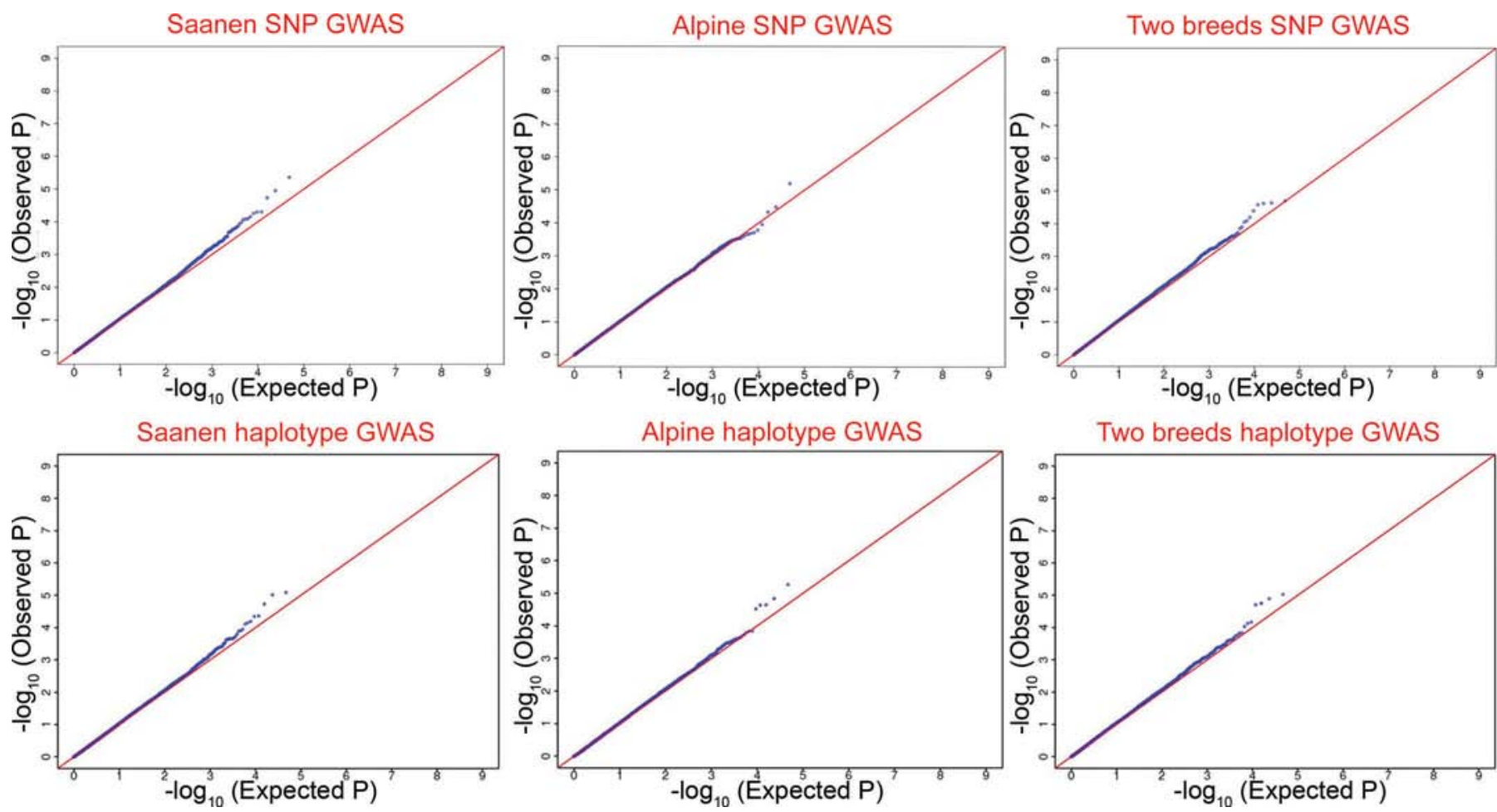

Figure 1. Quantile-quantile plots of the genome-wide association study analyses SNP by SNP and by haplotype for the analyses within and across the Saanen and the Alpine breeds. Color version available online. 


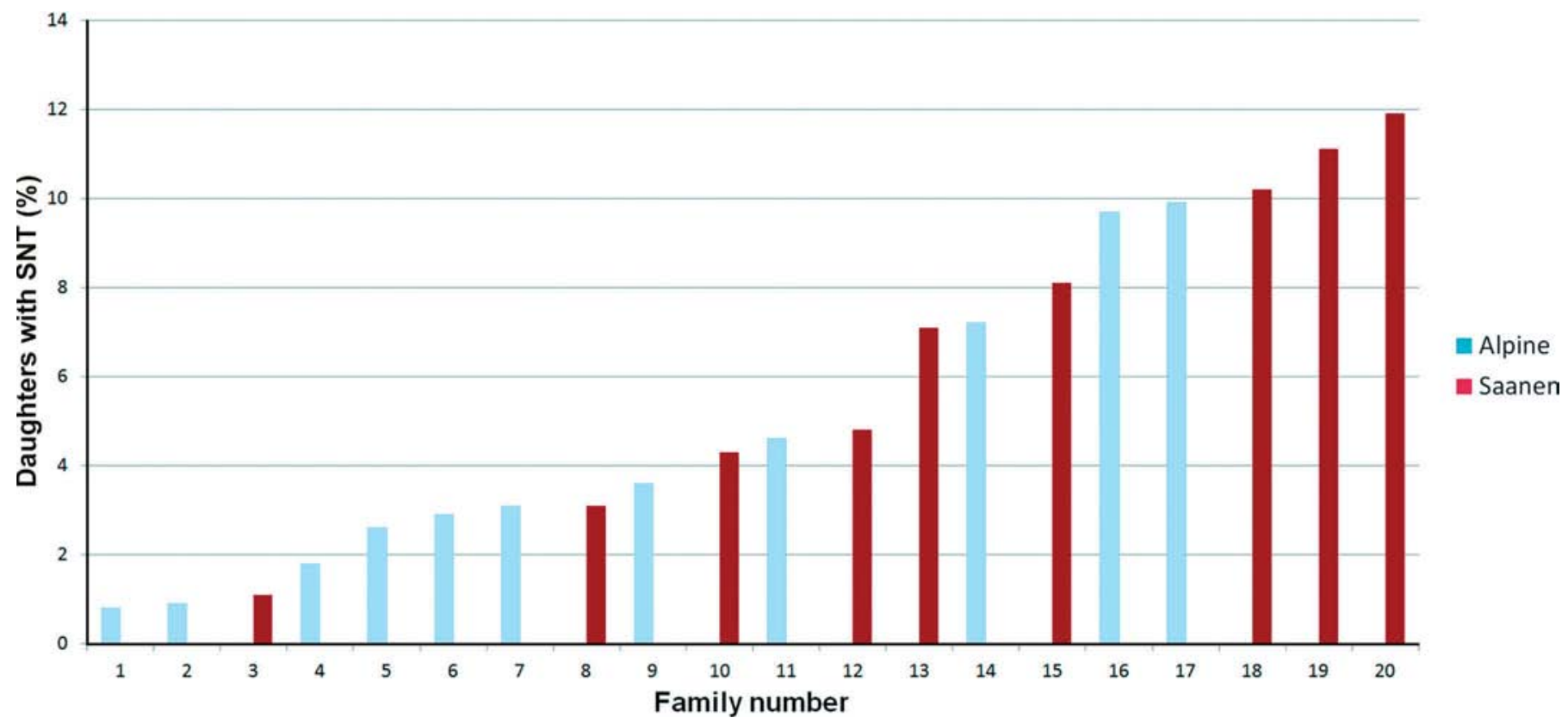

Figure 2. Distribution of the supernumerary teats frequencies in families from the genome-wide association study design. Each column corresponds to one family (all the daughters from the same sire) and the color of the bar indicates the breed: blue (light gray) for Alpine and red (black) for Saanen. Color version available online.

concluded that SNT exhibit substantial genetic variability, and that genetic selection and detection of the genomic regions controlling this phenotype might be feasible.
The GWAS analyses revealed a moderate number of signals scattered all over the goat genome (17 regions on 10 chromosomes), as illustrated in Figure 5, but none was above the genome significance threshold.

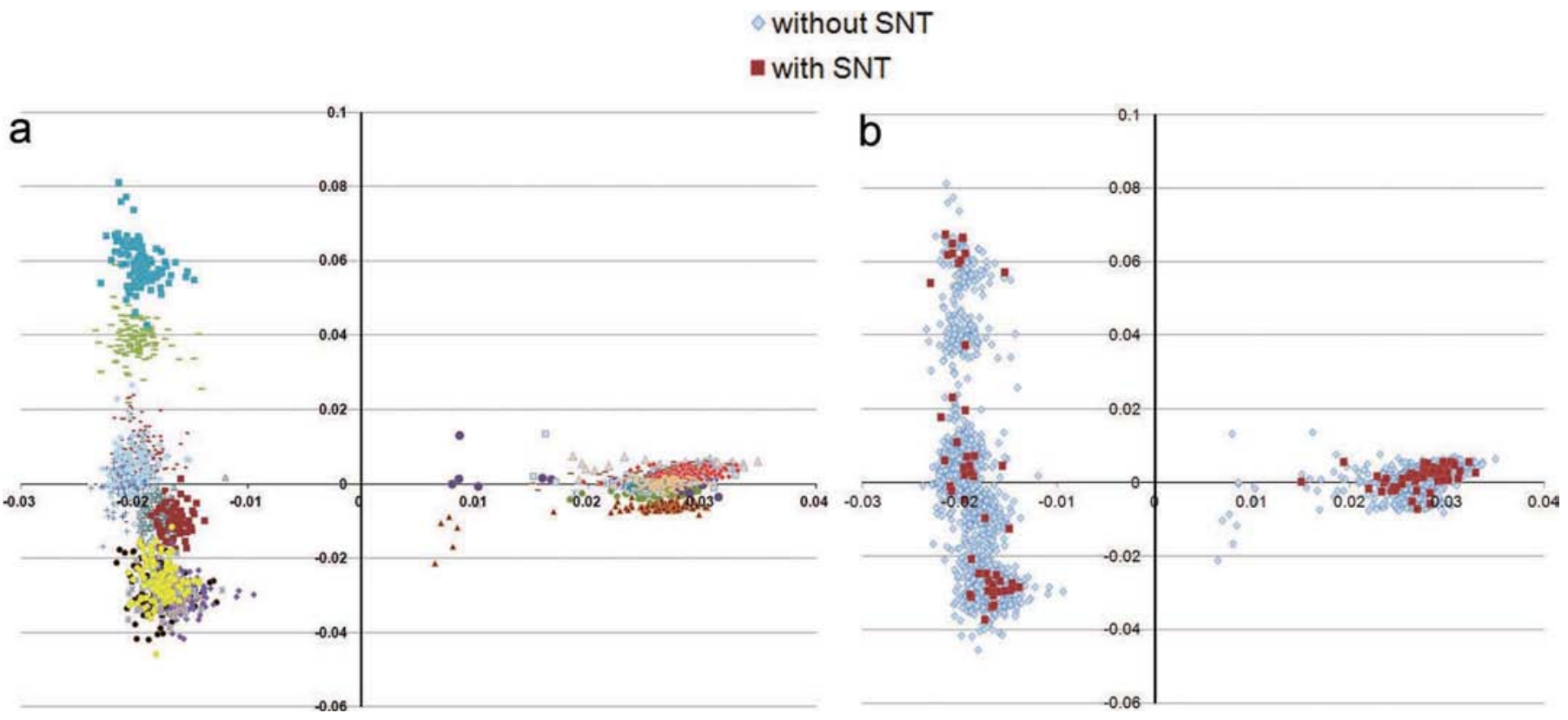

Figure 3. Population structure map drawn from the first 2 principal components (a) by family with each symbol corresponding to 1 family and (b) by phenotype. SNT = supernumerary teats. Color version available online. 
Thus, SNT presence seems to be a polygenic trait, and no evidence of segregation of a major gene was apparent in our Saanen and Alpine populations. Despite some evidence for dominant single-gene inheritance, based on pedigree or familial studies, in other mammalian species (Vainikainen, 1945; Goertzen and Ibsen, 1951; Urbani and Betti, 1996; Howard and Gusterson, 2000), the situation in goats seems to be different. Our results tend to agree with more recent analyses in cattle, based on familial (Brka et al., 2000) or genomic studies (Pausch et al., 2012; Joerg et al., 2014), which led to the conclusion that determinism was polygenic or oligogenic.

The absence of any overlap between the SNP detected by SNP and by haplotype-based analyses in the regions identified was striking, given that the animals were the same. One reason might be that the 2 methods detect different types of signal. Single nucleotide polymorphism GWAS is the best way to detect a signal (causal mutation) associated with a single SNP but in low linkage disequilibrium with its neighbors. Theoretically, however, this method may produce some false positives. In comparison, the GWAS by haplotype, which is based on phased haplotype clusters in association, accounts for the potential linkage disequilibrium between markers. Based on both simulated and real barley data, Lorenz et al. (2010) concluded that superiority of one over the other method to detect QTL depends on the population history and architecture of traits and recommended use of haplotype methods as a complement to SNP GWAS. Strengths of the SNP methods include the fact that errors in map order will have no effect on the single SNP analysis, but may lead to improper inference of haplotype alleles. Also, haplotype GWAS tests are based on chi-squared distributions with more degrees of freedom than for single
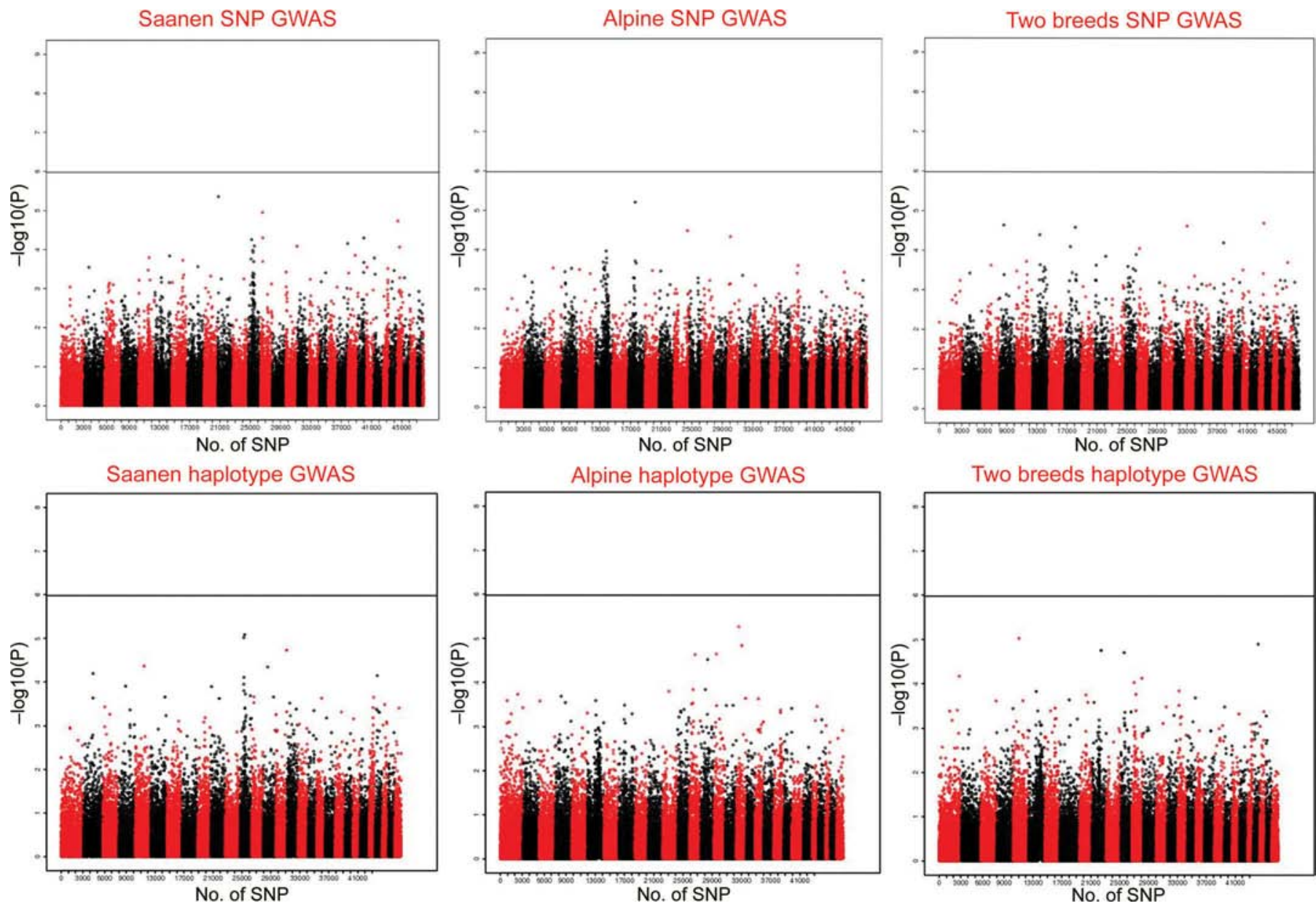

Figure 4. Genome-wide Manhattan plots of the genome-wide association study (GWAS) analyses SNP by SNP and by haplotype for the analyses within and across the Saanen and the Alpine breeds. Manhattan plots show the combined association $\operatorname{signals}_{5}\left[-\log _{10}(P)\right] \operatorname{on}$ the $\mathrm{y}$-axis versus SNP position in the goat genome on the x-axis and ordered by chromosome number. Black lines represent the $5 \%$ genome-wide threshold. Chromosomes are ordered from Capra hircus autosome (CHI)1 to CHI29 and the X chromosome is the last one. Color version available online. 


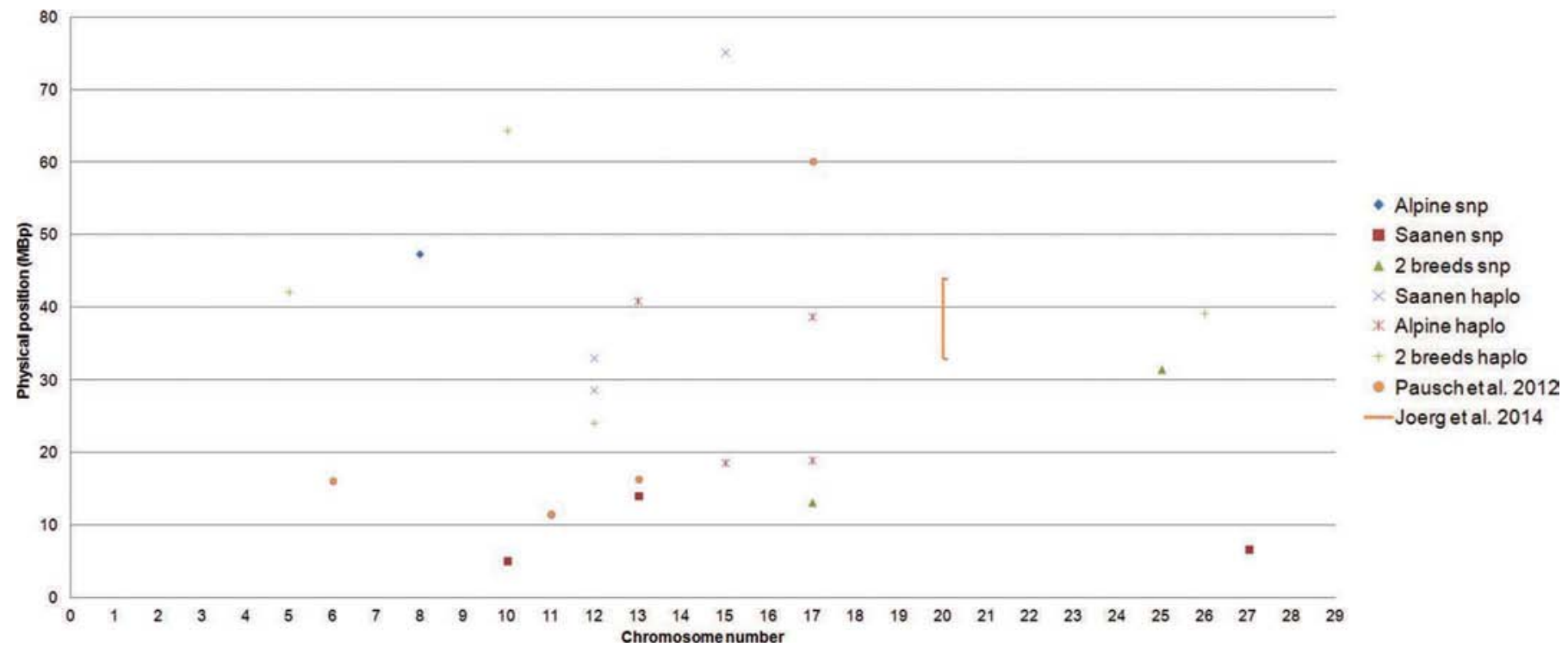

Figure 5. Map showing the position of all the chromosome-wide significant signals on the goat genome, with the chromosome number on the $\mathrm{x}$-axis and the physical position of the signal on the chromosome (in Mbp) on the $\mathrm{y}$-axis. In addition to our significant signals, the significant positions found in cattle are here placed on their corresponding position on the goat genome and labeled by their study name [Pausch el al. (2012) or Joerg et al. (2014)]. Color version available online.

SNP, so that for the same $P$-value the likelihood ratios need to be higher to reach the significance thresholds (Lorenz et al., 2010). However, an advantage of the multiplicity of alleles combinations in the haplotype GWAS is the increased likelihood of detecting a signal if the gene responsible is more than biallelic. Generally, the current haplotype-based association did not provide more hits or hits, with much smaller $P$-values than the SNP by SNP approach, but highlighted different genomic regions. Moreover, the robustness of the haplotype-based association is greatly affected by the accuracy of haplotyping reconstruction, which is dependent on the family structure of the sample and the parameters used. A major gene for SNT should have

Table 2. List of all the chromosome-wide significant signals from genome-wide association study SNP by SNP and by haplotype within and across the breeds ${ }^{1}$

\begin{tabular}{|c|c|c|c|c|}
\hline Population or method & $\mathrm{CHI}^{2}$ & $\begin{array}{l}\mathrm{SNP} \text { with highest } \\
P \text {-value }\end{array}$ & $\begin{array}{c}\text { Physical position } \\
\left(10^{6} \mathrm{bp}\right)\end{array}$ & $P$-value \\
\hline Two breeds haplotype & 5 & rs268265687 & 42.2 & $9.4 \times 10^{-6}$ \\
\hline Alpine SNP & 8 & rs268285073 & 47.3 & $6.2 \times 10^{-6}$ \\
\hline Saanen SNP & 10 & rs 268285073 & 5.0 & $4.4 \times 10^{-6}$ \\
\hline Two breeds haplotype & 10 & rs268250768 & 64.4 & $1.7 \times 10^{-5}$ \\
\hline Two breeds haplotype & 12 & rs 268281872 & 24.2 & $2.0 \times 10^{-5}$ \\
\hline Saanen haplotype & 12 & rs268262693 & 28.7 & $9.6 \times 10^{-6}$ \\
\hline Saanen haplotype & 12 & rs 268262693 & 33.2 & $8.2 \times 10^{-6}$ \\
\hline Saanen SNP & 13 & rs268287664 & 14.0 & $1.1 \times 10^{-5}$ \\
\hline Alpine haplotype & 13 & rs268238738 & 40.8 & $2.3 \times 10^{-5}$ \\
\hline Alpine haplotype & 15 & rs268258938 & 18.6 & $2.3 \times 10^{-5}$ \\
\hline Saanen haplotype & 15 & rs268246131 & 75.2 & $1.9 \times 10^{-5}$ \\
\hline Two breeds SNP & 17 & rs268248686 & 13.2 & $2.4 \times 10^{-5}$ \\
\hline Alpine haplotype & 17 & rs268253631 & 19.0 & $5.4 \times 10^{-6}$ \\
\hline Alpine haplotype & 17 & rs268284335 & 38.7 & $1.5 \times 10^{-5}$ \\
\hline Two breeds SNP & 25 & rs 268242456 & 31.5 & $2.0 \times 10^{-5}$ \\
\hline Two breeds haplotype & 26 & rs268252404 & 39.2 & $1.3 \times 10^{-5}$ \\
\hline Saanen SNP & 27 & rs268262482 & 6.7 & $1.9 \times 10^{-6}$ \\
\hline
\end{tabular}

${ }^{1}$ The table gives for each signal: the corresponding population and method; that is, SNP by SNP or by haplotype, the chromosome number, the SNP where the maximum is reached, and its position and the associated probability.

${ }^{2}$ Capra hircus autosome. 
been detected by both methods, or at least by one, but all the analyses pointed toward polygenic control with several loci with small $P$-values.

The loci associated with SNT were on different chromosomes or at different positions in the 2 breeds studied separately. These results seem to indicate that the main genes responsible for SNT - or allele frequencies - truly differ between the 2 breeds.

This observation is in accordance with previous QTL detections for other traits in these 2 breeds, using the same data set, which showed shared QTL locations across breeds only for some dairy traits and different genetic control for most of the other traits (Maroteau et al., 2013). Our aim in combining the 2 breeds was to increase the power and detect those loci that showed consistent association with SNT across the 2 breeds. This approach resulted mostly in the identification of new locations not previously found within the breed. Therefore, the SNP detected by the mixed breeds analyses are likely linked with shared loci that have a consistent but small effect on the SNT within each breed.

None of the regions detected here corresponded to the orthologous region of the bovine chromosome 20 associated with bovine SNT (Joerg et al., 2014), or to 3 of the 4 signals detected in the second study (Pausch et al., 2012). However, the signal located in an orthologous region on CHI13 (BTA5, 186,792 bp) matches an SNP detected for the Saanen breed in the SNP analysis. The associated region on the bovine genome contains predicted genes similar to genes that are known to be involved in Wnt signaling. This pathway could be involved in embryonic mammary gland development and is also a potential candidate for SNT formation (Pausch et al., 2012).

In our study, SNT information was expressed in a binary manner (i.e., presence or absence). However, a more accurate description of this phenotype would include the number of SNT, their position, and whether or not they are functional. At present, this information is not available. The phenotype we used in our study may be a mix of different traits and under the control of different genes, which might, to some extent, explain our confusing GWAS results. The results of Joerg et al. (2014) indicated a very high heritability (0.604) when only the caudal SNT were taken into account. Thus, although determinism seems to be polygenic in each case, heritability might be increased and selection more rapid if the phenotype could be better defined. In our study the phenotype was not defined in detail (we only had the presence or absence of SNT information), but developing this aspect could be a future goal.

\section{CONCLUSIONS}

The frequency $(4 \%)$ and heritability $(0.40-0.44)$ of the presence of SNT were estimated for the 2 major French goat breeds: Alpine and Saanen. The large amount of genotyping data enabled us to find multiple regions of the genome associated at chromosome-wide significance level with the trait for each breed. Only small signals were detected, leading us to hypothesize a polygenic determinism. These results imply that the possibility of a markers-based selection on the SNT trait is unlikely. However, as this trait is heritable, and routinely recorded, it could be managed with a dedicated EBV. At present, only animals with SNT are excluded from the assortative mating done within the framework of the breeding scheme, and the presence of relatives carrying SNT is not taken into account. The creation of an EBV, by providing better information about each animal, would be a better way of selecting against this trait and reducing the frequency of this undesirable phenotype.

\section{ACKNOWLEDGMENTS}

The authors thank Pierre Martin and all the Capgenes (Mignaloux Beauvoir, France) breeding organizations for the data provided and their helpful contributions. This work was supported by grants from French organizations through "Genomcap" [a research program including INRA, APIS-GENE, Union Nacionale des Coopératives d'Elevage et d'Insémination Animale (UNCEIA), Capgenes, and France Conseil Elevage (FCEL)] and EC (FP7/2007-2013), grant $\mathrm{n}^{\circ} 245140$, "3SR," Sustainable Solutions for Small Ruminants (http://www.3srbreeding.eu/). The authors thank the involved partners.

\section{REFERENCES}

Blowey, R. W., and A. D. Weaver. 2011. Udder and teat disorders. Pages 203-219 in Color Atlas of Diseases and Disorders of Cattle. 3rd ed. R. W. B. D. Weaver, ed. Mosby, Edinburgh, UK.

Brka, M., N. Reinsch, and E. Kalm. 2000. Determination of the inheritance pattern of hyperthelia in cattle by maximum likelihood analysis. J. Anim. Breed. Genet. 117:425-431. http://dx.doi. org/10.1046/j.1439-0388.2000.00273.x.

Brka, M., N. Reinsch, and E. Kalm. 2002. Frequency and Heritability of supernumerary teats in German Simmental and German Brown Swiss cows. J. Dairy Sci. 85:1881-1886. http://dx.doi.org/10.3168/ jds.S0022-0302(02)74262-8.

Brka, M., N. Reinsch, C. Tölü, and T. Savaş. 2007. Heritability of supernumerary teats in Turkish Saanen goats. Page 75 in 58th Annual Meeting of EAAP, Dublin, Ireland.

Browning, S. R., and B. L. Browning. 2007. Rapid and accurate haplotype phasing and missing-data inference for whole-genome association studies by use of localized haplotype clustering. Am. J. Hum. Genet. 81:1084-1097. http://dx.doi.org/10.1086/521987. 
Chalkias, H., L. Rydhmer, and N. Lundeheim. 2013. Genetic analysis of fuctional and non-functional teats in a population of Yorkshre pigs. Livest. Sci. 152:127-134.

Drickamer, L. C., T. L. Rosenthal, and R. D. Arthur. 1999. Factors affecting the number of teats in pigs. J. Reprod. Fertil. 115:97-100.

Druet, T., and M. Georges. 2010. A hidden Markov model combining linkage and linkage disequilibrium information for haplotype reconstruction and quantitative trait locus fine mapping. Genetics 184:789-798. http://dx.doi.org/10.1534/genetics.109.108431.

Gifford, W. 1934. The occurrence of polythelia in dairy cattle. J. Dairy Sci. 17:559-569. http://dx.doi.org/10.3168/jds.S00220302(34)93273-2.

Gilmour, A. R., B. J. Gogel, B. R. Cullis, and R. Thompson. 2009 ASReml User Guide Release 3.0. VSN International Ltd., Hemel Hempstead, UK.

Goertzen, B., and H. L. Ibsen. 1951. Supernumerary mammae in guinea pigs. J. Hered. 42:307-311.

Hirooka, H., D. J. de Koning, B. Harlizius, J. A. van Arendonk, A P. Rattink, M. A. Groenen, E. W. Brascamp, and H. Bovenhuis. 2001. A whole-genome scan for quantitative trait loci affecting teat number in pigs. J. Anim. Sci. 79:2320-2326.

Howard, B. A., and B. A. Gusterson. 2000. The characterization of a mouse mutant that displays abnormal mammary gland development. Mamm. Genome 11:234-237. http://dx.doi.org/10.1007/ s003350010043.

Joerg, H., C. Meili, O. Ruprecht, E. Bangerter, A. Burren, and A. Bigler. 2014. A genome-wide association study reveals a QTL influencing caudal supernumerary teats in Holstein cattle. Anim. Genet. 45:871-873. http://dx.doi.org/10.1111/age.12215.

Labussière, J. 1988. Review of physiological and anatomical factors influencing the milking ability of ewes and the organization of milking. Livest. Prod. Sci. 18:253-274. http://dx.doi.org/10.1016/03016226(88)90035-8.

Lorenz, A. J., M. T. Hamblin, and J.-L. Jannink. 2010. Performance of single nucleotide polymorphisms versus haplotypes for genomewide association analysis in barley. PLoS ONE 5:e14079 http:// dx.doi.org/10.1371/journal.pone.0014079.
Maroteau, C., I. Palhiere, H. Larroque, V. Clément, G. Tosser-Klopp, and R. Rupp. 2013. QTL detection for traits of interest for the dairy goat industry. Page 598 in 64th Annual Meeting of EAAP, Nantes, France.

Pausch, H., S. Jung, C. Edel, R. Emmerling, D. Krogmeier, K.-U. Götz, and R. Fries. 2012. Genome-wide association study uncovers four QTL predisposing to supernumerary teats in cattle. Anim. Genet. 43:689-695. http://dx.doi.org/10.1111/j.1365-2052.2012.02340.x.

Peris, S., X. Such, and G. Caja. 1996. Milkability of Murciano-Granadina dairy goats. Milk partitioning and flow rate during machine milking according to parity, prolificacy and mode of suckling. J. Dairy Res. 63:1-9.

Rowson, A. R., K. M. Daniels, S. E. Ellis, and R. C. Hovey. 2012 Growth and development of the mammary glands of livestock: A veritable barnyard of opportunities. Semin. Cell Dev. Biol. 23:557566. http://dx.doi.org/10.1016/j.semcdb.2012.03.018.

Tosser-Klopp, G., P. Bardou, O. Bouchez, C. Cabau, R. Crooijmans Y. Dong, C. Donnadieu-Tonon, A. Eggen, H. C. M. Heuven, S. Jamli, A. J. Jiken, C. Klopp, C. T. Lawley, J. McEwan, P. Martin, C. R. Moreno, P. Mulsant, I. Nabihoudine, E. Pailhoux, I. Palhière, R. Rupp, J. Sarry, B. L. Sayre, A. Tircazes, J. Wang, W. Wang, W. Zhang, and the International Goat Genome Consortium. 2014. Design and Characterization of a 52K SNP Chip for Goats. PLoS ONE 9:e86227. 10.1371/journal.pone.0086227.

Urbani, C. E., and R. Betti. 1996. Accesory mammary tissue in clinical practice. J. Eur. Acad. Dermatol. Venereol. 9:94.

Urbani, C. E., and R. Betti. 1998. Supernumerary nipple in association with Becker Nevus vs. Becker Nevus syndrome: A semantic problem only. Am. J. Med. Genet. 77:76-77.

Vainikainen, V. 1945. On the heritability of supernumerary nipples in Finnish home-bred sheep. J. Sci. Agric. Soc. Finl. 17:11-21.

Yang, J., S. H. Lee, M. E. Goddard, and P. M. Visscher. 2011. GCTA A tool for genome-wide complex trait analysis. Am. J. Hum. Genet. 88:76-82. http://dx.doi.org/10.1016/j.ajhg.2010.11.011. 